Check for updates

Cite this: Phys. Chem. Chem. Phys., 2019, 21, 57

Received 6th September 2018, Accepted 3rd October 2018

DOI: $10.1039 / c 8 c p 05662$

rsc.li/pccp

\section{Highly efficient surface hopping dynamics using a linear vibronic coupling model $\dagger$}

\author{
Felix Plasser, (iD *ab Sandra Gómez, ${ }^{\text {b }}$ Maximilian F. S. J. Menger, ${ }^{\text {bc }}$ Sebastian Mai (iD ${ }^{b}$ \\ and Leticia González (D) *b
}

\begin{abstract}
We report an implementation of the linear vibronic coupling (LVC) model within the surface hopping dynamics approach and present utilities for parameterizing this model in a blackbox fashion. This results in an extremely efficient method to obtain qualitative and even semi-quantitative information about the photodynamical behavior of a molecule, and provides a new route toward benchmarking the results of surface hopping computations. The merits and applicability of the method are demonstrated in a number of applications. First, the method is applied to the $\mathrm{SO}_{2}$ molecule showing that it is possible to compute its absorption spectrum beyond the Condon approximation, and that all the main features and timescales of previous on-the-fly dynamics simulations of intersystem crossing are reproduced while reducing the computational effort by three orders of magnitude. The dynamics results are benchmarked against exact wavepacket propagations on the same LVC potentials and against a variation of the electronic structure level. Four additional test cases are presented to exemplify the broader applicability of the model. The photodynamics of the isomeric adenine and 2-aminopurine molecules are studied and it is shown that the LVC model correctly predicts ultrafast decay in the former and an extended excited-state lifetime in the latter. Futhermore, the method correctly predicts ultrafast intersystem crossing in the modified nucleobase 2-thiocytosine and its absence in 5-azacytosine while it fails to describe the ultrafast internal conversion to the ground state in the latter.
\end{abstract}

\section{Introduction}

The trajectory surface hopping $\operatorname{method}^{1}$ is a powerful computational tool that allows for the study of quantum transitions occurring during ultrafast molecular photodynamical processes. Using this method, a variety of processes occurring within one spin multiplicity have been studied, such as the primary event in vision, ${ }^{2}$ photodeactivation of nucleobases ${ }^{3}$ and other biological chromophores, ${ }^{4}$ as well as photochemical organic reactions ${ }^{5}$ and photocatalysis. ${ }^{6}$ Even more, through an extension of this formalism to include spin-orbit coupling (SOC), ${ }^{7}$ it has been possible to elucidate intersystem crossing (ISC) processes in a variety of molecules, e.g. modified nucleobases,${ }^{8}$ nitroaromatics, ${ }^{9,10}$ and transition metal complexes. ${ }^{11}$ Notwithstanding their popularity, ${ }^{12-18}$ standard

\footnotetext{
${ }^{a}$ Department of Chemistry, Loughborough University, Loughborough, LE11 3TU, UK. E-mail: F.Plasser@lboro.ac.uk

${ }^{b}$ Institute for Theoretical Chemistry, Faculty of Chemistry, University of Vienna, Währingerstr.17, 1090 Vienna, Austria.E-mail: leticia.gonzalez@univie.ac.at ${ }^{c}$ Dipartimento di Chimica e Chimica Industriale, University of Pisa,

Via G. Moruzzi 13, 56124 Pisa, Italy

$\dagger$ Electronic supplementary information (ESI) available: Employed LVC parameters for $\mathrm{SO}_{2}$ (S1-S4) and details on the fitting procedures for Ade and 2AP (S5) (pdf); SHARC input files containing all LVC parameters and data underlying the presented figures (zip). See DOI: 10.1039/c8cp05662e
}

$a b$ initio surface hopping approaches suffer from two downsides, their high computational cost and the difficulty of verifying whether the approximations made when going from the quantum to the quasiclassical description are appropriate. The high computational cost stems from the $a b$ initio computations carried out at every time step of every trajectory, often requiring hundreds of thousands of such calculations for the whole ensemble. Because of this high cost, the simulation of large molecules, long-time dynamics, and rare events is often not feasible. Furthermore, the need of a large amount of on-the-fly computations often requires that cheaper but less accurate electronic structure methods are employed, possibly deteriorating the quality of the results.

Different strategies have been developed for reducing the number of electronic structure computations in trajectory dynamics simulations. These include a parameterization of the surfaces through interpolated diabatic Hamiltonians, ${ }^{19,20}$ on-the-fly constructed databases, ${ }^{21,22}$ or artificial neural networks. ${ }^{23,24}$ However, none of these approaches is in routine use, which probably derives from the fact that they do require a significant amount of electronic structure computations and expert knowledge to be applied successfully. Here, we want to proceed in a different way and combine the surface hopping method with a very popular approach that is in routine use and has been well-tested and refined by numerous groups over the 
last 30 years, the vibronic coupling (VC) model. ${ }^{25} \mathrm{VC}$ models provide a description of the main physics of interacting potential surfaces, including the conical shape of their intersections, using only a minimum number of parameters with clear physical meaning. They can be parameterized using standardized protocols ${ }^{25-29}$ and are commonly used in the context of quantum dynamics, in particular within the wellestablished multiconfigurational time-dependent Hartree (MCTDH) method, ${ }^{30}$ and have been shown to be powerful for describing ultrafast nonadiabatic processes in organic and inorganic molecules, ${ }^{31-34}$ in transition metal complexes ${ }^{27,35,36}$ and at interfaces. ${ }^{37}$ Despite the huge popularity of surface hopping and VC models individually, it is difficult to find any combined application of both methods in the literature, ${ }^{38}$ and certainly no generally applicable implementation is available. However, such a combination will be highly desirable as it allows speeding up surface hopping simulations by orders of magnitude when compared to on-the-fly dynamics and allows for including essentially an unlimited number of degrees of freedom as opposed to quantum dynamics. At the same time, it does not introduce any new approximation that is not already included in either one of the two well-tested constituent methods. If a particular photophysical problem can be described by both methods individually it should also be described correctly by the combined approach. Conversely, as we will discuss below, the method provides a convenient and versatile approach for evaluating the reliability of its different ingredients such as the electronic structure data, the surface hopping algorithm, and the parameterized potential energy surfaces. Finally, it offers a new route for evaluating the influence of different degrees of freedom in VC models used in quantum dynamics. For the above reasons, we deem a general implementation of LVC surface hopping highly desirable.

With this motivation in mind, we created a general interface for performing surface hopping dynamics using VC models within the SHARC (surface hopping with arbitrary couplings) molecular dynamics package. ${ }^{7,17,39,40}$ In this work, we investigate the simplest possible case, i.e., a VC model using only linear terms (LVC) parameterized using only a single excitedstate computation, and study its usefulness for addressing various photophysical problems. First, sulfur dioxide $\left(\mathrm{SO}_{2}\right)$ is studied, a molecule that has recently attracted significant attention, ${ }^{34,41-46}$ due to its ultrafast ISC occurring on a subpicosecond time scale. Here, we investigate whether the employed LVC model can predict the occurrence of ultrafast ISC and whether it correctly describes the participation of the different electronic states, a question that has been open since decades ${ }^{47}$ and was clarified only a few years ago. ${ }^{41,44}$ Second, adenine and its structural isomer 2-aminopurine (2AP) are investigated. The remarkable feature of these two molecules is that despite their structural similarity, they exhibit a completely different photophysical behavior. ${ }^{48,49}$ Adenine undergoes non-radiative decay on a subpicosecond time scale, ${ }^{50-54}$ whereas the closely related $2 \mathrm{AP}$ system possesses an extended excited state lifetime ( $>100 \mathrm{ps}$ ) in gas phase ${ }^{55}$ and is even fluorescent in many solvents. ${ }^{48,56}$ We examine whether the presented SHARC/LVC approach allows discriminating between these qualitatively different behaviours. As a third test case, the modified nucleobase 2-thiocytosine (2TC) is investigated. 2TC belongs to the class of thio-nucleobases, which have been the target of much research ${ }^{57-60}$ due to their interesting photophysical properties based on their remarkably ultrafast ISC. In 2TC, ISC occurs on a $200-400$ fs time scale, ${ }^{58}$ whereas virtually no decay to the $S_{0}$ occurs. Finally, we investigate 5 -azacytosine $(5 \mathrm{AC})$, which is the chromophore of the widespread anti-cancer drug azacytidine. ${ }^{61}$ As its mechanism of action involves incorporation into DNA, its photophysics is of interest regarding druginduced light sensitivity and the crucial feature is that it undergoes decay to the ground state rather than ISC. ${ }^{62,63}$

\section{Methods}

\subsection{Wavefunction representations}

For the following discussion, it is beneficial to briefly review the different possible representations of electronic wavefunctions and establish the naming conventions ${ }^{39,64}$ used in the rest of the paper, see Fig. 1. Standard quantum chemistry codes deal with an electronic Hamiltonian that includes molecular Coulomb interactions but neither external fields nor SOC. We label this operator the molecular Coulomb Hamiltonian $(\mathrm{MCH})$ and its eigenfunctions form the $\mathrm{MCH}$ representation (Fig. 1(b)). In the MCH representation, the states possess a distinct multiplicity and are labelled $S_{1}, S_{2}, \ldots, T_{1}, T_{2}, \ldots$ States of the same spin-multiplicity do not cross in a one-dimensional picture whereas states of different multiplicities do. The Hamiltonian including SOC is termed the "total Hamiltonian" and its eigenfunctions, generally possessing mixed spin, form the "diagonal" representation (Fig. 1(c)). These states do not cross in a one-dimensional picture and can be labelled with numbers $1,2, \ldots$, etc. An alternative way of transforming the $\mathrm{MCH}$ states is by minimizing nonadiabatic interactions leading to states of almost constant character in the diabatic representation (Fig. 1(a)). To indicate the diabatic representation, we either use symmetry labels $\left({ }^{1} \mathrm{~B}_{1},{ }^{1} \mathrm{~A}_{2}\right)$ or labels describing the state character $\left({ }^{1} \pi \pi^{*},{ }^{1} n \pi^{*}\right)$.

Per construction, the LVC model works in the diabatic basis. It is worth noting that MCTDH works entirely in the diabatic basis and can directly take the LVC model as input. In contrast,

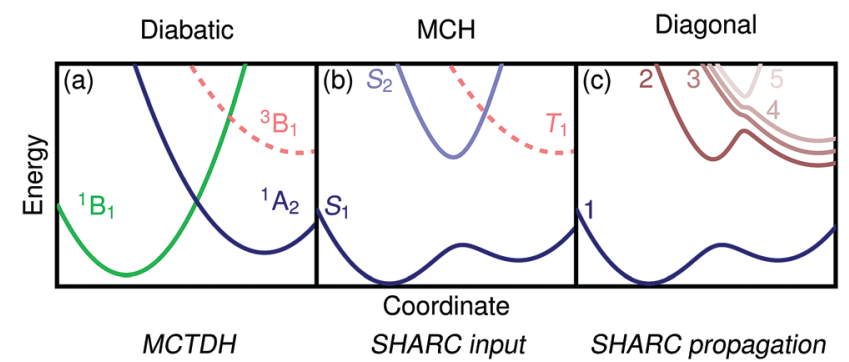

Fig. 1 Wavefunction representations used in this work: (a) the diabatic representation, which is the basis for the LVC model and used in the $\mathrm{MCTDH}$ dynamics, (b) the $\mathrm{MCH}$ representation, which is used in standard quantum chemical codes and is the input for SHARC, and (c) the diagonal representation, which is used during the SHARC dynamics. 
SHARC expects input in the MCH representation and propagates the wavefunction in the diagonal picture. It will be, thus, necessary to transform the input as described below (Section 2.5). The SHARC output can be given in any of the three pictures explained above, and it is, thus, possible to perform a one-to-one comparison with MCTDH despite the fact that different representations were used for the wavefunction propagation.

\subsection{The linear vibronic coupling model}

In a VC model the molecular Coulomb Hamiltonian operator, as defined above, is constructed in a diabatic representation as

$$
\mathbf{V}=V_{0} \mathbf{1}+\mathbf{W}
$$

where $V_{0}$ is the ground state potential and the $\mathbf{W}$ matrix collects the state-specific vibronic coupling terms. In the harmonic approximation, the ground state potential is given as

$$
V_{0}=\frac{1}{2} \mathbf{r}^{\mathrm{T}} \mathbf{H}_{0} \mathbf{r}
$$

where $\mathbf{r}$ is the displacement from the reference geometry in Cartesian coordinates and $\mathbf{H}_{0}$ is the ground state Hessian. To rewrite this equation, one first diagonalizes the mass-weighted Hessian

$$
\begin{gathered}
\sqrt{\mathbf{M}^{-1}} \mathbf{H}_{0} \sqrt{\mathbf{M}^{-1}}=\mathbf{K} \Omega^{2} \mathbf{K}^{\mathrm{T}} \\
\Omega^{2}=\operatorname{diag}\left({\omega_{1}}^{2},{\omega_{2}}^{2}, \ldots, \omega_{3 N}{ }^{2}\right),
\end{gathered}
$$

where $\mathbf{M}$ is the diagonal matrix containing the atomic masses $M_{\alpha}$, to obtain the normal-mode frequencies $\omega_{i}$ and the normal modes expressed in terms of mass-weighted coordinates (contained in the orthogonal matrix K). Insertion of eqn (3) into eqn (2) and rearranging the terms yields

$$
V_{0}=\frac{\hbar}{2} \underbrace{\mathbf{r}^{\mathrm{T}} \sqrt{\mathbf{M}} \mathbf{K} \sqrt{\frac{\Omega}{\hbar}}}_{\mathbf{Q}^{\mathrm{T}}} \Omega \underbrace{\sqrt{\frac{\Omega}{\hbar}} \mathbf{K}^{\mathrm{T}} \sqrt{\mathbf{M}} \mathbf{r}}_{\mathbf{Q}} .
$$

Here, the vector $\mathbf{Q}=\left(Q_{1}, \ldots, Q_{3 N}\right)^{\mathrm{T}}$ represents the displacement in terms of dimensionless mass-frequency scaled normal coordinates ( $c f$. ref. 29), explicitly given as

$$
Q_{i}=\sqrt{\frac{\omega_{i}}{\hbar}} \sum_{\alpha} K_{\alpha i} \sqrt{M_{\alpha}} r_{\alpha} .
$$

Using these coordinates, the harmonic ground state potential [eqn (2)] is given as

$$
V_{0}=\sum_{i} \frac{\hbar \omega_{i}}{2} Q_{i}^{2}
$$

Within the current work, an LVC model is considered, which contains the following state-specific terms in the $\mathbf{W}$ matrix.

$$
\begin{gathered}
W_{n n}=\varepsilon_{n}+\sum_{i} \kappa_{i}^{(n)} Q_{i} \\
W_{m n}=\sum_{i} \lambda_{i}^{(m, n)} Q_{i}
\end{gathered}
$$

Here the $\varepsilon_{n}$ are the vertical excitation energies, while the $\kappa_{i}^{(n)}$ and $\lambda_{i}^{(m, n)}$ are termed intrastate and interstate vibronic coupling constants. $^{25}$

\subsection{Parameterization}

In this work, we investigate the applicability of a "one-shot" LVC approach, i.e., using a parameterization that derives only from a single excited-state electronic structure computation. For this purpose, a new module was added to the SHARC molecular dynamics package ${ }^{17,40}$ that allows determining all parameters of the LVC model using only a ground-state frequency computation and a single-point calculation of excited-state energies, gradients, nonadiabatic couplings, and SOCs at the equilibrium geometry. The advantages of this module are that it works in a blackbox fashion, even in the case of many degrees of freedom, a high density of excited states, and the absence of symmetry. ${ }^{65}$

In the "one-shot" LVC approach, the $\omega_{i}$ values are simply the ground state vibrational frequencies while the $\varepsilon_{n}$ energies and diabatic SOC constants are the vertical excitation energies and SOCs at the equilibrium geometry, respectively. The intrastate vibronic coupling constants are computed as the derivative of the electronic energy $E_{n}$ of state $n$ with respect to a normal mode $^{25,27}$ computed at the reference geometry, which using eqn (6) can be rearranged as

$$
\kappa_{i}^{(n)}=\frac{\partial E_{n}}{\partial Q_{i}}=\sqrt{\frac{\hbar}{\omega_{i}}} \sum_{\alpha} \frac{\partial E_{n}}{\partial r_{\alpha}} \frac{K_{\alpha i}}{\sqrt{M_{\alpha}}}
$$

where $\partial E_{n} / \partial r_{\alpha}$ is the gradient in Cartesian coordinates. The offdiagonal elements are defined as matrix elements of the derivative of the electronic Hamiltonian $\hat{H}^{\mathrm{MCH}}$ evaluated with respect to a normal mode displacement ${ }^{29}$

$$
\begin{aligned}
\lambda_{i}^{(m n)} & =\left\langle\Psi_{m}^{\mathrm{MCH}}\left|\frac{\partial \hat{H}^{\mathrm{MCH}}}{\partial Q_{i}}\right| \Psi_{n}^{\mathrm{MCH}}\right\rangle \\
& =\sqrt{\frac{\hbar}{\omega_{i}}} \sum_{\alpha}\left\langle\Psi_{m}^{\mathrm{MCH}}\left|\frac{\partial \hat{H}^{\mathrm{MCH}}}{\partial r_{\alpha}}\right| \Psi_{n}^{\mathrm{MCH}}\right\rangle \frac{K_{\alpha i}}{\sqrt{M_{\alpha}}}
\end{aligned}
$$

where $\Psi_{m}^{\mathrm{MCH}}$ and $\Psi_{n}^{\mathrm{MCH}}$ are the $\mathrm{MCH}$ eigenfunctions determined at the reference geometry.

Commonly, the $\lambda$ parameters are evaluated indirectly using energy-based information only, by considering either the excited state Hessian ${ }^{25,27,34}$ or by fitting the potential energy surfaces to model potentials. ${ }^{28,29}$ However, following arguments by Yarkony and coworkers, ${ }^{66}$ it is possible to evaluate eqn (11) directly and this approach is used here. In the case of configuration interaction (CI) computations, the $\left\langle\Psi_{m}^{\mathrm{MCH}}\left|\partial \hat{H}^{\mathrm{MCH}} / \partial r_{\alpha}\right| \Psi_{n}^{\mathrm{MCH}}\right\rangle$ terms can be obtained from derivatives of the CI-matrix yielding a quantity termed $\mathbf{f}^{\mathrm{CI}}$ that is closely related to the nonadiabatic coupling vector. ${ }^{66,67}$ Similar equations have also been incorporated within coupled cluster theory. ${ }^{68,69}$ In cases where nonadiabatic coupling vectors are not available, it is possible to compute the $\lambda_{i}^{(m n)}$ values in the same spirit through a numerical differentiation using a recently described algorithm ${ }^{65}$ based on wavefunction overlaps. $^{70}$ 
An LVC model, even when constructed in this simple way, provides qualitatively correct potential energy surfaces in the vicinity of the expansion point and assures the proper conical topology of intersections between adiabatic states. However, the quality of the description is expected to deteriorate if the molecule undergoes large-amplitude structural changes, such that anharmonicities and higher-order vibronic coupling terms become important. As a consequence, it is not expected that the presented protocol is adequate in describing all photochemical reactions or other processes involving strong structural rearrangements. In these cases, it may be possible to resort to higher-order VC models and more sophisticated parameterization schemes ${ }^{26,28}$ or on-the-fly dynamics will have to be applied. On the other hand, we expect that the simple "one-shot" LVC approach can provide a qualitatively correct description of photophysical processes, like internal conversion and ISC, that are dominated by the electron dynamics and do not involve the breaking or creation of chemical bonds or the motion of flexible groups. In Section 3, we investigate the applicability of this method for the description of a number of realistic photophysical processes and find remarkably good agreement with experiment as well as with more expensive computational protocols.

\subsection{SHARC dynamics}

The SHARC method, used for propagating dynamics on coupled surfaces of different multiplicity has been recently reviewed in ref. 17. For completeness, we provide here a brief overview. The nuclei follow classical trajectories obtained by integrating Newton's equation:

$$
M_{\alpha} \frac{\partial^{2} r_{\alpha}}{\partial t^{2}}=-\mathbf{g}_{m}^{\text {diag }}
$$

where the term on the right is the negative gradient of the electronic energy of the current active electronic state. The active electronic state is determined by the surface hopping procedure. To this end, the total electronic wave function

$$
\left|\Phi^{\mathrm{el}}(t)\right\rangle=\sum_{m} c_{m}(t)\left|\Psi_{m}^{\mathrm{diag}}\right\rangle
$$

expressed through the electronic coefficients $c_{m}(t)$, is propagated in time concordantly with the nuclear trajectory. Here, within the SHARC approach the basis states $\Psi_{m}^{\text {diag }}$ are chosen to be the eigenstates of the total electronic Hamiltonian $\hat{H}^{\text {total }}=\hat{H}^{\mathrm{MCH}}+\hat{H}^{\prime}$. Here, $\hat{H}^{\prime}$ collects all the terms that go beyond the $\mathrm{MCH}$, e.g., SOC or couplings to an external field. The eigenstates are constructed by a unitary transformation of the states coming from the electronic structure computation:

$$
\left|\Psi_{m}^{\mathrm{diag}}\right\rangle=\sum_{n}\left|\Psi_{n}^{\mathrm{MCH}}\right\rangle U_{n m}
$$

The transformation matrix $\mathbf{U}$ is computed by the diagonalization

$$
\mathbf{H}^{\text {diag }}=\mathbf{U}^{\dagger} \mathbf{H}^{\mathrm{MCH}} \mathbf{U},
$$

where $\mathbf{H}^{\mathrm{MCH}}$ is the matrix representation of the total Hamiltonian within the $\mathrm{MCH}$ basis, i.e., $H_{m n}^{\mathrm{MCH}}=\left\langle\Psi_{m}^{\mathrm{MCH}}\left|\hat{H}^{\text {total }}\right| \Psi_{n}^{\mathrm{MCH}}\right\rangle$.
Practically, with the above diagonalization carried out, the coefficients $c_{m}(t)$ can be propagated according to the matrix equation

$$
\mathbf{c}(t+\Delta t)=\underbrace{\mathbf{U}^{\dagger}(t+\Delta t) \mathbf{P}^{\mathrm{MCH}}(t+\Delta t, t) \mathbf{U}(t)}_{\mathbf{P}(t+\Delta t, t)} \mathbf{c}(t),
$$

where $\mathbf{P}^{\mathrm{MCH}}(t+\Delta t, t)$ is the propagator matrix in the $\mathrm{MCH}$ representation, which can be computed, e.g., using the local diabatization approach. ${ }^{71}$ Once the new coefficients $\mathbf{c}(t+\Delta t)$ are known, the hopping probabilities can be computed

$$
h_{n \rightarrow m}=\left(1-\frac{\left|c_{n}(t+\Delta t)\right|^{2}}{\left|c_{n}(t)\right|^{2}}\right) \frac{\operatorname{Re}\left[c_{m}(t+\Delta t) P_{m n}{ }^{*} c_{n}{ }^{*}(t)\right]}{\left|c_{n}(t)\right|^{2}-\operatorname{Re}\left[c_{n}(t+\Delta t) P_{n n}{ }^{*} c_{n}{ }^{*}(t)\right]} .
$$

and the active state is found stochastically.

In this work, the gradient $\mathbf{g}_{m}^{\text {diag }}$ of the diagonal state, needed to propagate eqn (12), is approximated as a linear combination of the MCH gradients

$$
\mathbf{g}_{m}^{\mathrm{diag}}=\sum_{n}\left|U_{n m}\right|^{2} \mathbf{g}_{n}^{\mathrm{MCH}} .
$$

Within SHARC, it is generally also possible to include an additional correction to the gradient involving the nonadiabatic coupling terms. ${ }^{39}$

As in other surface hopping approaches, after a successful hop the nuclear momenta are adjusted to conserve total energy and by default the full velocity vector is rescaled. Furthermore, the electronic coefficients are adjusted after each time step to consider decoherence; here we use the well-established energybased decoherence correction introduced by Granucci et al. ${ }^{72}$

\subsection{Interface to SHARC}

All quantities required by the SHARC dynamics program can be constructed from the LVC model using the workflow sketched in Fig. 2. First, the normal mode displacements are computed from the Cartesian geometry according to eqn (6). Then, the potential matrix $\mathbf{V}$ is computed using eqn (7)-(9) and subsequently diagonalized according to

$$
\mathbf{V T}=\mathbf{T} \operatorname{diag}\left(E_{1}, \ldots, E_{N_{\mathrm{st}}}\right)
$$

where $\mathbf{T}$ is the diabatic-to-MCH transformation matrix and $E_{n}$ are the $\mathrm{MCH}$ energies. Gradients and nonadiabatic couplings are computed by taking the derivatives of eqn (7)-(9) with respect to normal mode displacement and subsequently transforming them to the $\mathrm{MCH}$ basis using the $\mathbf{T}$ matrix and to Cartesian coordinates in analogy to eqn (6). In addition, SOCs and dipole moments can be converted from the diabatic to the $\mathrm{MCH}$ basis using the T matrix. Wavefunction overlaps between the MCH states at two successive dynamics time steps, which are needed for propagation using the local diabatization formalism, ${ }^{71,73}$ are evaluated according to

$$
\mathbf{S}(t, t+\Delta t)=\mathbf{T}(t)^{\mathbf{T}} \mathbf{T}(t+\Delta t) .
$$

In summary, the new interface provides all quantities in the $\mathrm{MCH}$ basis and in Cartesian coordinates, so that for the SHARC 


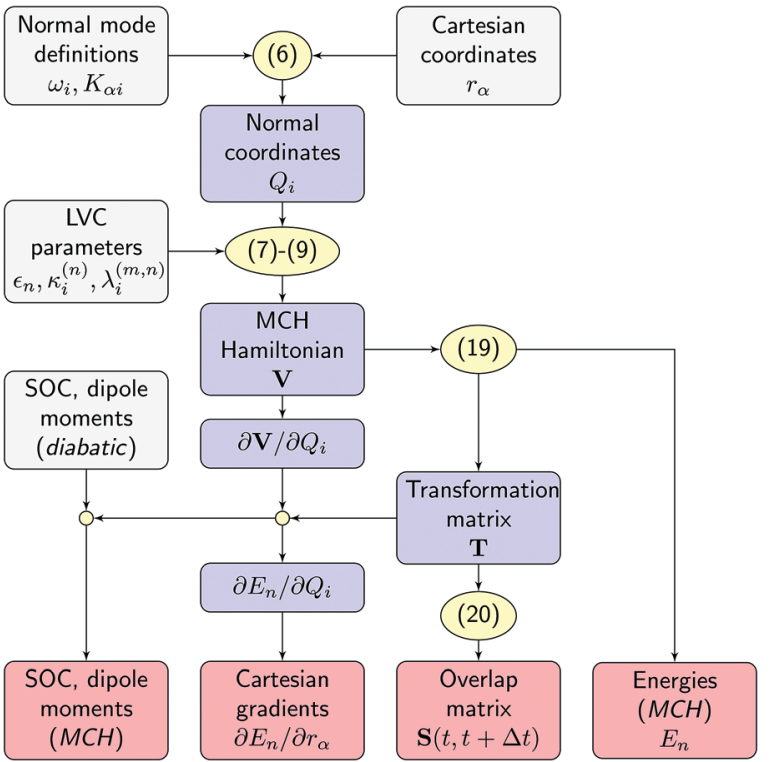

Fig. 2 Workflow of the SHARC/LVC method. Input quantities, intermediates and output are given in grey, blue, and red boxes, respectively. Equation numbers are shown in yellow.

dynamics driver the simulations do not differ from on-the-fly simulations. This also means that all analysis procedures implemented in SHARC, considering, for example, Cartesian coordinates and electronic populations, can automatically be applied to the LVC-based simulations.

Technically speaking, in our original implementation the timedetermining step was not the evaluation of the matrix operations but the communication between the Fortran SHARC driver and the LVC program written in Python. Therefore, a new driver for SHARC called PySHARC - was developed within the course of this project, utilizing Python's C API (application program interface), thus allowing for in-memory communication between the Python interface and SHARC's Fortran routines, replacing the file based communication used so far. Additionally, the new implementation makes it possible to read all parameters just once and store them in memory reducing the number of file operations to be performed and enhancing efficiency even further.

\subsection{Computational details}

Electronic structure computations on $\mathrm{SO}_{2}$ were carried out at two different levels of theory: (i) multireference configuration interaction (MR-CI) ${ }^{74}$ including single excitations using a complete active space of 6 active electrons in 6 orbitals as reference space, and a polarized double- $\zeta$ basis set of ANO-RCC ${ }^{75}$ type [MR-CIS(6,6)/VDZP] following ref. 43, and (ii) MR-CI with single and double excitations using a larger $(12,9)$ active space and a polarized triple- $\zeta$ basis set ${ }^{75}$ [MR-CISD(12,9)/VTZP]. In both cases, the orbitals were generated at the complete-active space self-consistent field level considering 12 electrons in 9 orbitals, CASSCF(12,9). Scalar relativistic effects were taken into account by using the secondorder Douglas-Kroll-Hess Hamiltonian ${ }^{76}$ while SOC was included through atomic mean-field integrals ${ }^{77,78}$ and MR-CI SOC values were computed in a perturbative fashion. ${ }^{79}$ The $\kappa$ parameters were computed from analytical MR-CI gradients ${ }^{80}$ and the $\lambda$ parameters were computed through derivatives of CI matrix elements. ${ }^{67}$ In the case of adenine and 2AP, the MR-CIS computations were performed using an active space of 10 electrons in 8 orbitals $(2 \times n$, $\left.3 \times \pi, 3 \times \pi^{*}\right)$ and the aug-cc-pVDZ ${ }^{81}$ basis set. In the case of 2 TC an active space of 10 electrons in 8 orbitals $\left(1 \times n, 4 \times \pi, 3 \times \pi^{*}\right)$ was used for generating the orbitals with CASSCF while a $(6,5)$ reference space was used for MR-CIS. For 5AC orbitals were generated using $\operatorname{CASSCF}(12,9)\left(2 \times \mathrm{n}, 4 \times \pi, 3 \times \pi^{*}\right)$ and again a $(6,5)$ reference space was used for MR-CIS. In both cases the cc-pVDZ basis $\operatorname{set}^{81}$ was used. MR-CI computations were performed with the COLUMBUS program system ${ }^{82-84}$ using integrals
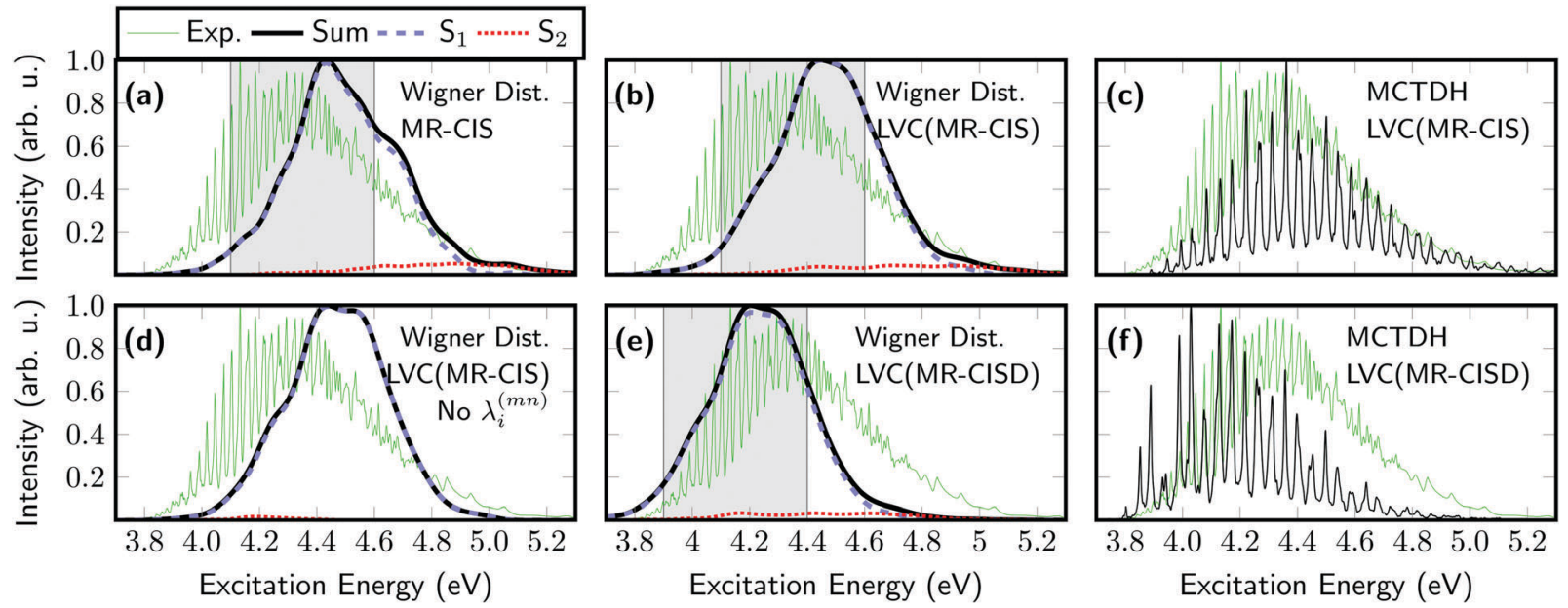

Fig. 3 Absorption spectrum of $\mathrm{SO}_{2}$ computed using six different computational protocols: sampling of the Wigner distribution using (a) 1000 individual MR-CIS(6,6)/VDZP computations and LVC models constructed at the (b) MR-CIS(6,6)/VDZP and (e) MR-CISD(12,9)/VTZP levels; spectra computed from MCTDH dynamics using the (c) LVC(MR-CIS) and (f) LVC(MR-CISD) models. For comparison, the LVC(MR-CIS) spectrum without interstate coupling parameters is shown in (d). The excitation windows used for initial condition generation in the surface hopping simulations are marked as grey boxes in (a), (b), and (e). 
generated with $\mathrm{MOLCAS}^{85}$ for $\mathrm{SO}_{2}, 2 \mathrm{TC}$, and $5 \mathrm{AC}$ and with DALTON $^{86}$ for adenine and 2AP.

The optical absorption spectrum of $\mathrm{SO}_{2}$ was computed according to a Wigner distribution of the ground state zero-point vibrational wavefunction, ${ }^{87}$ using normal modes computed at MR-CIS level. The surface hopping dynamics simulations on $\mathrm{SO}_{2}$ were started according to the excitation windows indicated as grey rectangles in Fig. 3 (4.1-4.6 eV for MR-CIS and 3.9-4.4 eV for MR-CISD). 4 singlet and 3 triplets states were considered in the dynamics and 200 trajectories, each, were propagated on the MR-CIS and MR-CISD potentials. A time step length of $0.5 \mathrm{fs}$ and a locally diabatic method for wavefunction propagation were used. ${ }^{71}$ An energy-based decoherence correction $(C=0.1 \mathrm{H})$ was used. ${ }^{72}$ For MCTDH,${ }^{30}$ the vibrational ground state wavefunction was promoted to the diabatic ${ }^{1} \mathrm{~B}_{1}$ state and the dynamics was propagated for $700 \mathrm{fs}$ using 10 single particle functions per normal mode, each expressed through 32 Legendre polynomials. UV absorption spectra were computed as the Fourier transform of the autocorrelation function obtained from the MCTDH dynamics and shifted by the zero-point energy of $0.1915 \mathrm{eV}$.

In the case of adenine the excitation window was chosen as $6.1 \pm 0.1 \mathrm{eV}$ and 209 trajectories were propagated for $1000 \mathrm{fs}$ considering 5 singlet states. For $2 \mathrm{AP}$ the excitation window was chosen as $5.0 \pm 0.1 \mathrm{eV}$ and 163 trajectories were propagated for $50 \mathrm{ps}$ considering 5 singlet states. The other set-up parameters (e.g., time step, propagator, decoherence correction) were kept as in the previous case. The normal modes used to construct the LVC model and to create the Wigner distribution were obtained at the RI-MP2/aug-cc-pVDZ level of theory using TURBOMOLE. ${ }^{88}$ For adenine and 2AP, relaxation time constants for the $S_{2} \rightarrow S_{1}$ and $S_{1} \rightarrow S_{0}$ processes were obtained by fitting a sequential first-order kinetics model to the population data. Errors of the time constants were obtained with the bootstrapping method, ${ }^{89}$ using 1000 bootstrapping samples for each ensemble (see S5 in the ESI $\nmid$ for more details on the fitting procedure). Note that these errors only describe the uncertainty due to the finite size of the trajectory ensembles but they make no statement about errors due to the electronic structure and dynamics methods.

The excitation windows chosen for 2TC and 5AC were $4.0 \pm$ $0.1 \mathrm{eV}$ and $5.0 \pm 0.2 \mathrm{eV}$, respectively, and in both cases 200 trajectories were computed. The normal modes were computed at the respective MR-CIS levels.

\section{Results and discussion}

\subsection{Sulfur dioxide}

The new method is exemplified first for $\mathrm{SO}_{2}$, for which the absorption spectrum as well as ultrafast ISC dynamics are investigated. The triatomic $\mathrm{SO}_{2}$ molecule is a convenient test case, as high level on-the-fly dynamics ${ }^{43}$ as well as full-dimensional quantum dynamics ${ }^{44}$ have been performed and can be used as a reference. Table 1 presents the vertical excitation energies computed for the equilibrium geometry for the MR-CIS(6,6)/VDZP and
Table 1 Vertical excitation energies (eV) and oscillator strengths (in parentheses) computed for $\mathrm{SO}_{2}$ at the MR-CIS(6,6)/VDZP and MRCISD(12,9)/VTZP levels of theory

\begin{tabular}{llll}
\hline & State & MR-CIS & MR-CISD \\
\hline $\mathrm{S}_{1}$ & ${ }^{1} \mathrm{~B}_{1}$ & $4.463(0.0029)$ & $4.227(0.0055)$ \\
$\mathrm{S}_{2}$ & ${ }^{1} \mathrm{~A}_{2}$ & 4.848 & 4.595 \\
$\mathrm{~S}_{3}$ & ${ }^{1} \mathrm{~B}_{2}$ & $6.805(0.0931)$ & - \\
$\mathrm{T}_{1}$ & ${ }^{3} \mathrm{~B}_{1}$ & 3.650 & 3.350 \\
$\mathrm{~T}_{2}$ & ${ }^{3} \mathrm{~B}_{2}$ & 4.478 & 4.211 \\
$\mathrm{~T}_{3}$ & ${ }^{3} \mathrm{~A}_{2}$ & 4.627 & 4.356 \\
\hline
\end{tabular}

MR-CISD(12,9)/VTZP levels of theory (see Computational details). The MR-CIS level shows that the $\mathrm{S}_{1}$ state $\left({ }^{1} \mathrm{~B}_{1}\right)$ located at $4.46 \mathrm{eV}$ is the only state below $6 \mathrm{eV}$ possessing oscillator strength. The $\mathrm{S}_{2}$ state $\left({ }^{1} \mathrm{~A}_{2}\right)$ is slightly higher in energy at $4.85 \mathrm{eV}$ and symmetryforbidden. The $S_{3}$ state $\left({ }^{1} B_{2}\right)$ is well-separated and lies close to $7 \mathrm{eV}$. The $T_{1}$ state $\left({ }^{3} B_{1}\right)$ is significantly lower in energy than any singlet state while $T_{2}\left({ }^{3} B_{2}\right)$ and $T_{3}\left({ }^{3} A_{2}\right)$ are energetically close to the $S_{1}$ and $\mathrm{S}_{2}$ states. The ordering of the states at the MR-CISD level agrees well with that of MR-CIS but the MR-CISD values are consistently down-shifted by $0.2-0.3 \mathrm{eV}$.

Next, optical absorption spectra were computed for $\mathrm{SO}_{2}$. The original spectrum, computed from 1000 individual MR-CIS computations and initially reported in ref. 43, is shown in Fig. 3(a). This spectrum is somewhat blue-shifted with respect to the experiment ${ }^{90}$ (green line) but the spectral width is approximately reproduced. Next, the spectrum was recomputed using the LVC model constructed at the MR-CIS level [Fig. 3(b)]. As explained above, all parameters for this model (see Tables S1-S4, ESI $\dagger$ ) were extracted from one single-point calculation at the equilibrium geometry. Remarkably, the LVC spectrum reproduces the full $a b$ initio result very well in terms of location of the peak, its width, and the amount of contribution of the $\mathrm{S}_{2}$ state, with a fraction of the computational effort. For comparison, the absorption spectrum was also computed using the MCTDH method. This spectrum, shown in Fig. 3(c), exhibits a fine structure that cannot be obtained with semiclassical methods, but otherwise it agrees on the position and overall broadening. Employing the LVC model allows us to compute the spectrum also at the computationally much more expensive MR-CISD level, as presented in Fig. 3(e). This spectrum is redshifted with respect to MR-CIS by about $0.2 \mathrm{eV}$, in agreement with Table 1, but otherwise the spectral shape is very similar. The MCTDH spectrum computed at the LVC(MR-CISD) level is presented in Fig. 3(f) showing a somewhat altered fine structure when compared to the MCTDH/LVC(MR-CIS) spectrum.

An important observation regarding the spectra presented above is that the adiabatic $S_{2}$ state gains some intensity and contributes to the spectrum at higher energies. This feature clearly violates the Condon approximation as the $S_{2}$ state is dark at the equilibrium geometry and, therefore, illustrates that the present protocol is able to compute spectra going beyond the Condon approximation. Hence, it is interesting to compare the spectra shown above with an LVC model that ignores the interstate couplings $\lambda_{l}^{(m n)}$. This corresponds to an approximation termed "vertical gradient", ${ }^{91}$ meaning that the 
ground and excited state frequencies are the same, the oscillator strengths are constant, and only gradient information is used to construct the spectrum. This spectrum is shown in Fig. 3(d) and resembles that obtained using the full LVC model [Fig. 3(b)] with the exception that the high-energy shoulder deriving from the adiabatic $\mathrm{S}_{2}$ state cannot be reproduced.

As a next step, nonadiabatic dynamics simulations considering 4 singlet and 3 triplet states were performed for five of the computational protocols introduced in Fig. 3. These correspond to SHARC dynamics at the on-the-fly MR-CIS, LVC(MR-CIS), and LVC(MR-CISD) levels, as well as MCTDH simulations at the LVC(MR-CIS) and LVC(MR-CISD) levels. Here, the difference in computational effort is noteworthy: while the 111 on-the-fly MR-CIS trajectories from ref. 43 propagated for 700 fs required about 15000 core hours on a modern CPU, the parameterization and all 200 trajectories at the LVC(MR-CIS) level were finished in about 6 core hours using the PySHARC implementation. To be able to compare results between SHARC and MCTDH we use the diabatic (spectroscopic) representation, ${ }^{39}$ i.e., we expand the timedependent electronic wavefunctions in terms of the electronic states of the symmetric equilibrium geometry using a procedure described in more detail in ref. 43. The results of the on-the-fly SHARC simulations at the MR-CIS level, originally reported in ref. 43, are shown in Fig. 4(a). The initial excitation goes predominantly to the ${ }^{1} B_{1}$ state, which is the $S_{1}$ state at the Franck-Condon geometry and the only symmetry-allowed state in the employed excitation window. Subsequently, the excited molecule stays on the $\mathrm{S}_{1}$ surface but an ultrafast change in diabatic character to ${ }^{1} \mathrm{~A}_{2}$ occurs making ${ }^{1} \mathrm{~A}_{2}$ the dominant state character already after 10 fs. Population of the triplet states is also ultrafast and after 700 fs the total triplet population amounts to $55 \%$. The LVC model [Fig. 4(b)] reproduces the main features of the on-the-fly simulations. The initial inversion between the ${ }^{1} \mathrm{~B}_{1}$ and ${ }^{1} \mathrm{~A}_{2}$ characters occurs at exactly $10 \mathrm{fs}$. Sub-picosecond triplet population is observed as well, where most population is received by the ${ }^{3} \mathrm{~B}_{2}$ state, in agreement with ref. 43 and 44. However, ISC is somewhat slower than in the on-the-fly calculations, and after 700 fs only 38\% triplet population is reached. The reduced population of the triplet states can be explained by the fact that ISC is most efficient during strong elongation of the $\mathrm{S}=\mathrm{O}$ bonds, ${ }^{43}$ which is difficult to describe using LVC potentials. Nonetheless, it is remarkable that the dynamics of $\mathrm{SO}_{2}$ including all the involved states and timescales can be qualitatively described by the LVC model, especially considering that the nature of the involved states was still under dispute until few years ago. ${ }^{41,44}$

The LVC method also directly allows judging the impact of quantum effects that cannot be captured by the surface hopping method. For this purpose quantum dynamics using the MCTDH method have been carried out for the LVC(MR-CIS) model, see Fig. 4(c). The results are very similar to the corresponding SHARC simulations [Fig. 4(b)] with the exception that stronger and more persistent oscillations between the singlet states are observed for $\mathrm{MCTDH}$ and that the triplet yield is somewhat lowered (only 27\% after $700 \mathrm{fs}$ ).

A further advantage of the SHARC/LVC method is that it is possible to directly investigate the effects of different parameters and algorithmic choices in the surface hopping method to see whether they affect the agreement between SHARC and MCTDH. To exemplify this, we changed one algorithmic detail in the SHARC dynamics. In many SHARC applications, to conserve energy after a surface hop, typically the full velocity vector is rescaled. If nonadiabatic coupling vectors are available, a more rigorous alternative ${ }^{1,16}$ is to rescale only the component of the velocity parallel to the nonadiabatic coupling
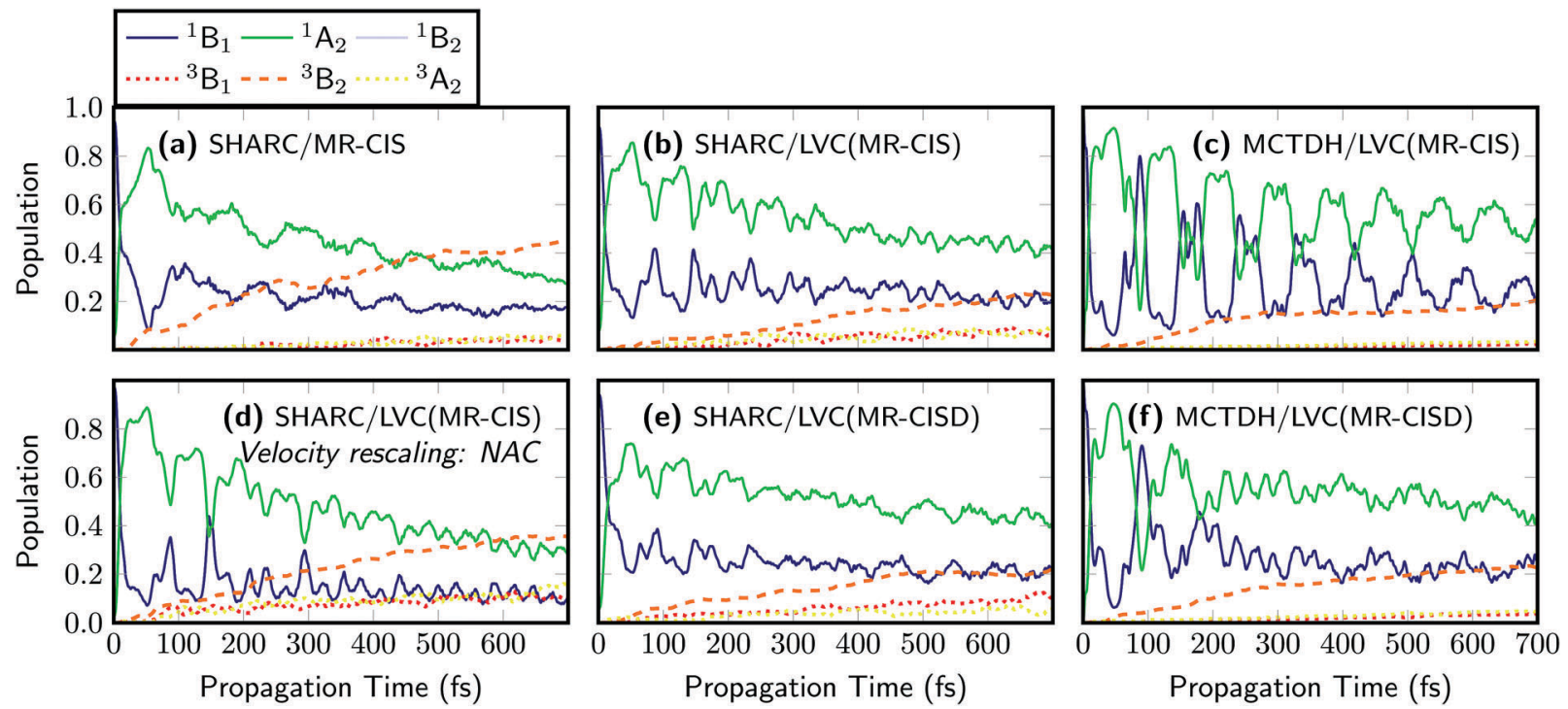

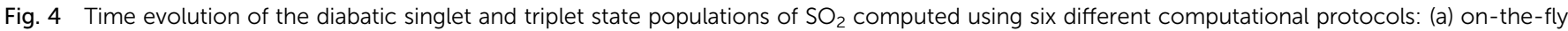

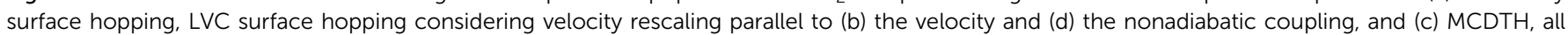
based on MR-CIS(6,6)/VDZP potentials; (e) LVC surface hopping and (f) MCTDH, both based on MR-CISD(12,9)/VTZP potentials. 
vector that induced the hop. The results of dynamics using this procedure are presented in Fig. 4(d). The different mode of rescaling leaves the qualitative picture unchanged but does affect the quantitative outcomes quite strongly. In particular, the triplet population after $700 \mathrm{fs}$ is raised to $61 \%$, which is twice as high as the MCTDH reference for the LVC(MR-CIS) potentials shown in Fig. 4(c). In addition, the oscillations between the singlet states are somewhat enhanced. It is noteworthy that the surface hopping results are so strongly affected by a seemingly innocuous algorithmic choice such as the mode of velocity rescaling. In the future, it will be thus of significant interest to evaluate the influence of other similar choices such as the treatment of frustrated hops and different options for decoherence corrections. The SHARC/LVC protocol provides an ideal way to perform such an evaluation for realistic highdimensional systems, and a more detailed comparison of SHARC and MCTDH results is currently in progress in our group.

The efficiency of the LVC method allows for a significant improvement of the computational level in terms of the excitation level, active space, and one-electron basis set to perform dynamics at the MR-CISD(12,9)/VTZP level [Fig. 4(e)], which is not feasible for on-the-fly dynamics. One can see that at this level of theory, the ${ }^{1} \mathrm{~B}_{1} /{ }^{1} \mathrm{~A}_{2}$ inversion happens somewhat slower and the populations are equal only after 15 fs owing to the fact that the magnitude of the $\lambda$ value coupling these two states is reduced from $0.20 \mathrm{eV}$ to $0.15 \mathrm{eV}$ ( $c f$. Tables $\mathrm{S} 1$ and $\mathrm{S} 2, \mathrm{ESI} \dagger$ ). Otherwise the dynamics is very similar, giving $38 \%$ triplet population after 700 fs. The MCTDH results computed using the LVC(MR-CISD) potentials are presented in Fig. 4(f). In this case, the ${ }^{1} \mathrm{~B}_{1} /{ }^{1} \mathrm{~A}_{2}$ inversion occurs after 11.5 fs and the triplet yield after 700 fs amounts to $31 \%$.

The MCTDH results shown here also compare well with the results of Lévêque et al. who used a more extended model Hamiltonian. ${ }^{44}$ In ref. 44 a similar oscillatory behaviour and a triplet yield of about $30 \%$ after 700 fs is reported. The main difference is that more persistent oscillations are observed in the present MCTDH/LVC(MR-CIS) dynamics. In a more general sense, the similarity of panels (b), (c), (e), and (f) suggests that neither the electronic structure level nor quantum effects play a decisive role in terms of the timescales or the states involved. On the other hand, a comparison with panel (a) shows that the inclusion of realistic on-the-fly potentials does have a notable effect on the triplet yield.

\subsection{Adenine and 2-aminopurine}

To evaluate the general applicability of the SHARC/LVC method to larger molecular systems, the adenine and 2AP molecules are investigated. In this case, it is of particular interest to investigate whether it is possible to correctly predict the qualitative differences in the photophysics of these two molecules, i.e., that adenine undergoes subpicosecond non-radiative decay ${ }^{50-54}$ whereas the closely related 2AP system possesses a more extended excited state lifetime $(>100 \mathrm{ps}) .^{48,55,56}$ The MR-CIS vertical excitation energies for adenine and 2AP are presented in Table 2. The results obtained for adenine are similar to previous studies employing the MR-CIS method. ${ }^{52,92}$ The first
Table 2 Vertical excitation energies (eV) and oscillator strengths (given in parentheses) of $9 \mathrm{H}$-adenine and $9 \mathrm{H}$-2-aminopurine computed at the MR-CIS(10/8)/aug-cc-pVDZ level of theory

\begin{tabular}{lllll}
\hline & State & Ade & State & 2 AP \\
\hline $\mathrm{S}_{1}$ & $1^{1} \mathrm{n} \pi^{*}$ & $5.677(0.000)$ & $1^{1} \mathrm{n} \pi^{*}$ & $4.782(0.003)$ \\
$\mathrm{S}_{2}$ & $1^{1} \pi \pi^{*}$ & $6.142(0.329)$ & $1^{1} \pi \pi^{*}$ & $4.991(0.112)$ \\
$\mathrm{S}_{3}$ & $2^{1} \pi \pi^{*}$ & $6.422(0.055)$ & $2^{1} \mathrm{n} \pi^{*}$ & $5.956(0.001)$ \\
$\mathrm{S}_{4}$ & $2^{1} \mathrm{n} \pi^{*}$ & $6.764(0.006)$ & $2^{1} \pi \pi^{*}$ & $6.590(0.231)$
\end{tabular}

singlet state, slightly below $6 \mathrm{eV}$ is of $\mathrm{n} \pi^{*}$ character. Two $\pi \pi^{*}$ states follow above $6 \mathrm{eV}$, the bright ${ }^{1} \mathrm{~L}_{a}$ state and the weakly absorbing ${ }^{1} \mathrm{~L}_{b}$ state, and $\mathrm{S}_{4}$ is again of $\mathrm{n} \pi^{*}$ character. These energies are consistently about $1 \mathrm{eV}$ above the experimental and computational gas phase reference values, ${ }^{49,93}$ but as the shift is systematic and in view of the successful applicability of previous MR-CIS simulations to describe the photodynamics of adenine,$^{52,92}$ we rely confidently on the suitability of the method. In Table 2 also the vertical excitation energies of 2AP are shown. The first excited states lie at lower energies than in the case of adenine and two states below $5 \mathrm{eV}$ are present, possessing $\mathrm{n} \pi^{*}$ and $\pi \pi^{*}$ character. The lower onset of absorption in 2AP is consistent with previous studies with the exception that the $\pi \pi^{*}$ state is usually considered to be the lowest state. $^{49,94,95}$

In a next step, the ultrafast dynamics after UV absorption are investigated for adenine and 2AP using the LVC(MR-CIS) model. The results for adenine are shown in Fig. 5(a). First, there is an ultrafast rise of the $\mathrm{S}_{1}$ state population, reaching a maximum value of $93 \%$ after 74 fs followed by a somewhat slower decay to $S_{0}$. Both features, the initial rise of $S_{1}$ and its subsequent decay agree remarkably well with on-the-fly MR-CIS dynamics, reported previously. ${ }^{52}$ A similar sub-100 fs process in the excited state manifold has also been observed with a quadratic coupling model in reduced dimensionality. ${ }^{96}$ The obtained relaxation time constants are $31 \pm 2$ fs and $1070 \pm$ $100 \mathrm{fs}$ for $\mathrm{S}_{2} \rightarrow \mathrm{S}_{1}$ and $\mathrm{S}_{1} \rightarrow \mathrm{S}_{0}$, respectively. These time constants agree very well with the experimental values of $<100 \mathrm{fs}$ and 700-1030 fs. ${ }^{50,51}$ At this point it is worth mentioning that the ultrafast nonradiative decay of adenine cannot be reproduced by time-dependent density functional theory (TDDFT) using a range of different popular density functionals. ${ }^{92,97}$ This shows that it is clearly a challenging feat to describe the process correctly and shows that an LVC model can even outperform on-the-fly TDDFT dynamics. While we do not claim that the SHARC/LVC protocol will always perform perfectly, it is fair to say that often it may be preferable to use an LVC model parameterized against a high-level reference instead of using a lower level method on-the-fly.

It is especially interesting to study whether the LVC model can describe the geometrical displacements leading to the $\mathrm{S}_{1} / \mathrm{S}_{0}$ decay, which are characterized by strong out-of-plane distortions of the aromatic rings. ${ }^{52,53,97}$ To this end, we show in Fig. 6 four exemplary $\mathrm{S}_{1} / \mathrm{S}_{0}$ hopping geometries from the LVC(MR-CIS) simulations. Indeed, the LVC model can predict these strong out-ofplane deformations, even though the LVC model is expanded around the almost perfectly planar $\mathrm{S}_{0}$ minimum. This is primarily achieved through non-zero out-of-plane $\lambda$ values between the 

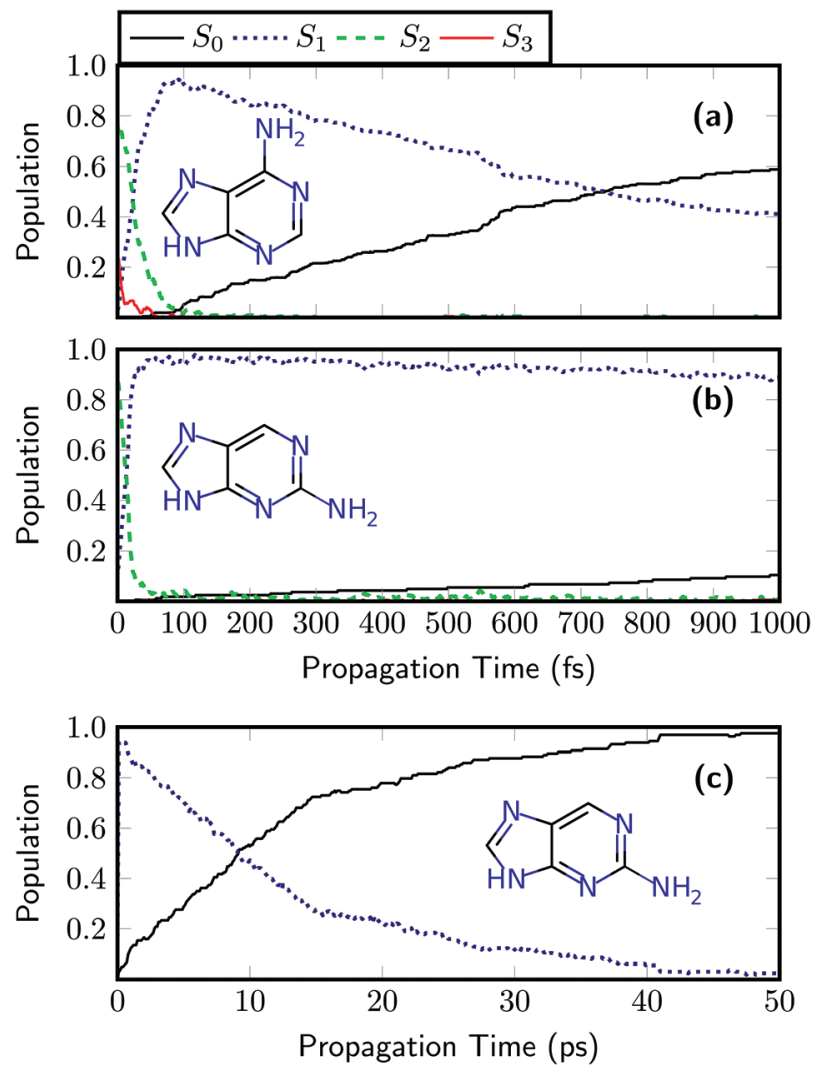

Fig. 5 Time evolution of the adiabatic singlet state populations of (a) $9 \mathrm{H}$ adenine and (b) $9 \mathrm{H}$-2-aminopurine after photoexcitation computed using LVC surface hopping based on MR-CIS(10,8)/aug-cc-pVDZ potentials. In panel (c) the evolution until $50 \mathrm{ps}$ is presented for $9 \mathrm{H}$-2-aminopurine.

diabatic $2^{1} n \pi^{*}$ and $2^{1} \pi \pi^{*}$ states. Consequently, at the hopping geometries the adiabatic $S_{1}$ state is a linear combination of the $2^{1} n \pi^{*}$ and $2^{1} \pi \pi^{*}$ states, while the adiabatic $S_{0}$ state is mostly of closed-shell character (with a weight $>70 \%$ ).

The dynamics results for 2AP are presented in Fig. 5(b). Also in this case an ultrafast rise of $S_{1}$ occurs, and an $S_{1}$ population of $92 \%$ is reached after only 32 fs. When compared to adenine, the subsequent decay to the ground state is significantly slowed down and after 1 ps, a ground state population of only $10 \%$ is reached, highlighting the enhanced nonradiative lifetime of 2AP. From Fig. 5(b) alone, it is challenging to obtain an

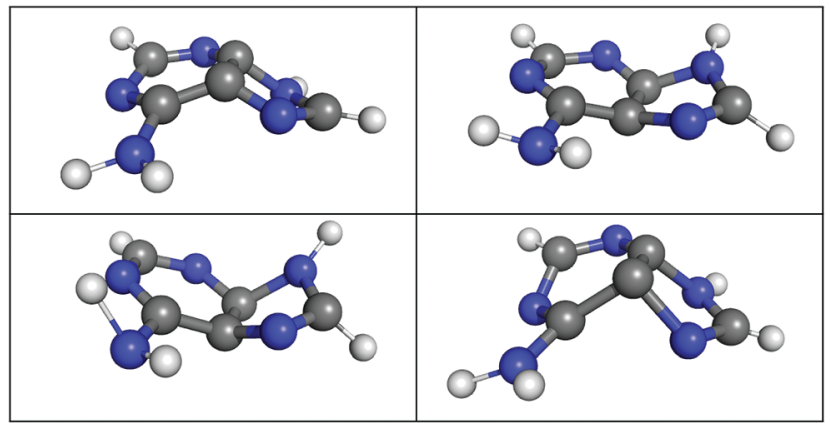

Fig. 6 Selected $\mathrm{S}_{1} / \mathrm{S}_{0}$ hopping geometries as obtained during LVC(MR-CIS) surface hopping dynamics of adenine. accurate estimate of the excited state lifetime. Therefore, the dynamics simulations were extended up to $50 \mathrm{ps}$. Such dynamics simulations, requiring over 16 million electronic structure computations for the whole ensemble, are far beyond the scope of $a b$ initio on-the-fly dynamics but can be easily performed with the LVC model. The results, presented in Fig. 5(c), reveal that after $9.0 \mathrm{ps}, 50 \%$ of the population has decayed to the ground state. The fitted time constants for $2 \mathrm{AP}$ are $17 \pm 1 \mathrm{fs}$ and $13000 \pm 1000$ fs for the $S_{2} \rightarrow S_{1}$ and $S_{1} \rightarrow S_{0}$ processes. The latter timescale is about one order of magnitude faster than the experimental value of 156 ps reported for jetcooled $2 \mathrm{AP} .{ }^{55}$ The main source for this error is probably the description of the low frequency out-of-plane modes. These are expected to experience non-negligible anharmonicities, which are not described in the LVC model. In order to definitely answer the question whether this problem is related to the LVC approximation, the level of theory MR-CIS, or the surface hopping protocol one would have to perform $a b$ initio MR-CIS dynamics, which are unfortunately unfeasible for the required timescale. Nonetheless, the employed LVC model correctly predicts two important properties of gas phase 2AP: that its lifetime is significantly longer than the one of adenine, and that it nevertheless decays on a picosecond timescale.

\section{3-Thiocytosine and 5-azacytosine}

To investigate the more general applicability for the new protocol, we finish this work by briefly studying two modified nucleobases. As a first example, we study 2-thiocytosine, i.e., keto-cytosine where one of the oxygen atoms is replaced by sulfur. The on-the-fly results for 2TC are presented in Fig. 7(a). ${ }^{58}$ Using the employed excitation window the dynamics starts predominantly in the $\mathrm{S}_{2}$ state. $\mathrm{S}_{2}$ decays on a time scale of about $160 \mathrm{fs}$, and the population is transferred to the $T_{1}$ and $S_{1}$ states with minor contributions from $T_{2}$ and $S_{2}$. Ultrafast ISC occurs with a time constant of $250 \mathrm{fs}^{58}$ At the end of the simulated period, the dominant state is $\mathrm{T}_{1}$ with a population of $58 \%$ and a total triplet yield of $75 \%$ is obtained. The main features of the dynamics, i.e. the $\mathrm{S}_{2}$ decay and the ultrafast ISC are well-captured by the SHARC/LVC dynamics shown in Fig. 7(b). Similarly to $\mathrm{SO}_{2}$ the main difference is that SHARC/ LVC somewhat underestimates the $\mathrm{T}_{1}$ population and the overall triplet yield, which is only $56 \%$ at the end of the simulated period. In general, the good performance of the LVC model for 2TC can be ascribed to the rigidity of the system in its excited state. ${ }^{58}$

As a final example, we want to discuss the 5-azacytosine molecule. The on-the-fly results ${ }^{63}$ for $5 \mathrm{AC}$ are presented in Fig. 8(a). The initial excitation is distributed over the $\mathrm{S}_{2}$ and $S_{1}$. A very rapid rise of $S_{1}$ follows and after about 100 fs almost all the population is in $S_{1}$. Around the same time, the rise of $S_{0}$ starts and after $600 \mathrm{fs}$ about half the population has transferred to $\mathrm{S}_{0}$. Triplet population is negligible. The SHARC/LVC dynamics [Fig. 8(b)] reproduces the initial ultrafast transfer to $\mathrm{S}_{1}$ and the absence of ISC in this system, and generally provides a good reproduction of the first 100 fs. However, it was not possible to reproduce the ultrafast decay to the ground state for 5AC. A likely reason for this behaviour is the presence of a 

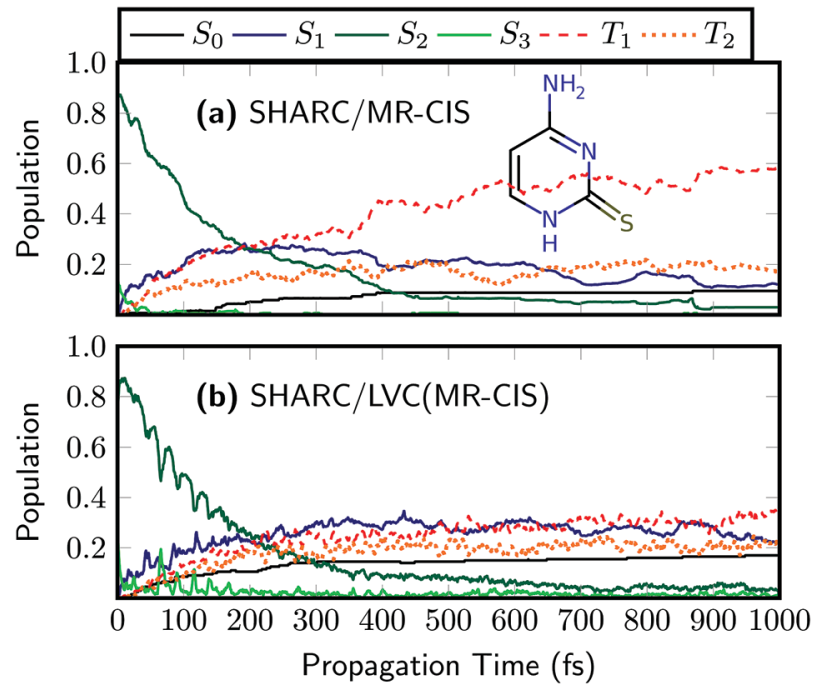

Fig. 7 Time evolution of the adiabatic populations of 2-thiocytosine after photoexcitation computed at the (a) on-the-fly and (b) LVC levels in SHARC using MR-CIS(6,5)/cc-pVDZ.
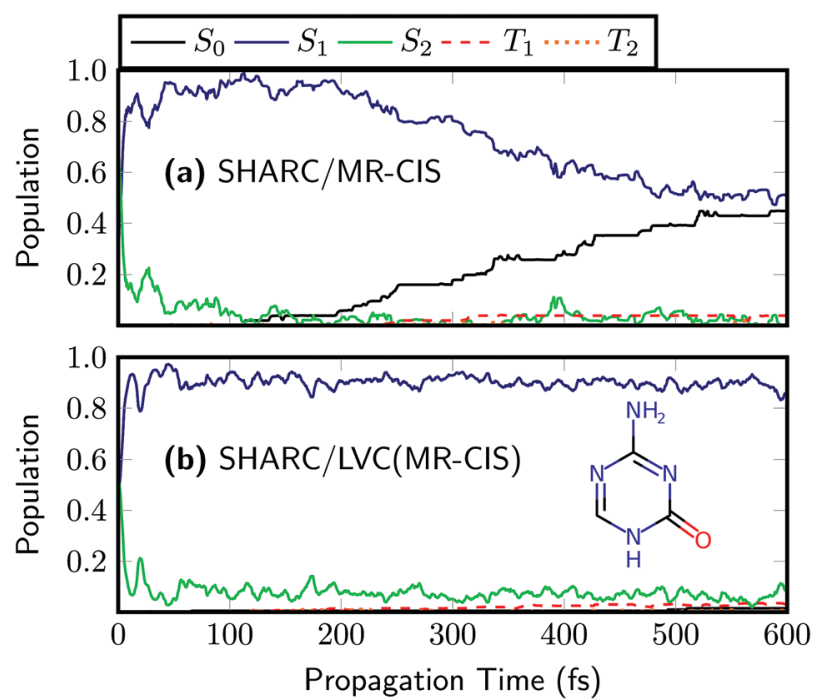

Fig. 8 Time evolution of the adiabatic populations of 5-azacytosine after photoexcitation computed at the (a) on-the-fly and (b) LVC levels in SHARC using MR-CIS(6,5)/cc-pVDZ.

barrier in the reaction path leading to the minimum energy conical intersection between $S_{1}$ and $S_{0} \cdot{ }^{63}$ In order to reproduce this barrier correctly, it is probably necessary to move beyond the LVC model.

\section{Conclusions}

A new implementation of the LVC model was reported, amenable for performing nonadiabatic trajectory surface hopping simulations and for constructing post-Condon spectra using a Wigner distribution formalism requiring only minimal computational and human effort. Utilities were developed that allow a parameterization of the model from only a ground-state frequency computation and one excited-state single-point computation. It was shown that despite being a simple and blackbox approach the method provides a powerful tool for addressing a variety of photophysical as well as methodological questions.

In the case of $\mathrm{SO}_{2}$, it was shown that the LVC model could reproduce all the features of the on-the-fly MR-CIS dynamics simulations while reducing the computational cost by about three orders of magnitude. It was also illustrated that the LVC model could be applied to evaluate the effects of the significantly enhanced MR-CISD level of electronic structure theory. Finally, because of the fact that the LVC model could be employed identically in quantum dynamics and surface hopping simulations, it provided a rigorous route for benchmarking the latter for realistic Hamiltonians of high-dimensional systems. Conversely, the method could, in the future, be used in the context of quantum dynamics studies, as it allows to efficiently evaluate the effect of neglecting different degrees of freedom.

An investigation of the prototypical adenine and 2AP molecules showed that the SHARC/LVC method allows to correctly discriminate between the different qualitative behavior of these two molecules: subpicosecond decay was found in the former whereas a significantly enhanced nonradiative lifetime was found in the latter. These results suggest that in cases where the LVC approximation is expected to work the method can be used as a blackbox and computationally cheap approach to evaluate whether or not a molecule is expected to be fluorescent - a notoriously difficult task in computational photochemistry.

Further tests were performed in 2TC and 5AC. In the case of 2TC, ultrafast decay of $S_{2}$ and ISC was found in agreement with on-the-fly dynamics. For 5AC some qualitative features of the on-the-fly dynamics were reproduced but the SHARC/LVC protocol failed to describe ultrafast decay to the ground state. The case of 5AC serves as a reminder that the "one shot" LVC approach investigated here is only the lowest level of a hierarchy of possibilities to approximate on-the-fly dynamics and will not always provide a definite answer. Nonetheless, it is impressive that the correct qualitative behaviour was found in four out of the five molecules studied considering the simplicity and computational efficiency of the approach.

It was shown that SHARC/LVC allows for a speed up of about three orders of magnitude compared to on-the-fly dynamics. This means that one can study one thousand molecules instead of one, a nanosecond instead of a picosecond, or an ensemble of 100000 trajectories instead of 100 allowing to study even very rare processes. Due to this low computational cost and its ease of use, we expect the presented protocol to be a powerful addition to the currently available computational photochemistry toolbox. We believe that it is a significant advancement towards black-box nonadiabatic dynamics methods, which could find applications in high-throughput screening for different purposes, e.g., fluorescent dyes or photostable drug molecules. At the same time caution is certainly in place. The presented protocol arises from the dire need of performing ab initio photodynamics simulations without incurring excessive computational costs. Whenever on-the-fly dynamics simulations are feasible at the same electronic 
structure level these are to be preferred over LVC dynamics and if quantum dynamics are possible, these are to be preferred over LVC surface hopping. Conversely, the protocol presents an attractive option for cases where ab initio on-the-fly dynamics is too expensive or the number of degrees of freedom is too large for quantum dynamics. We have used a "one shot" LVC approach, where all excited-state data is obtained from a single electronic structure computation, to show that even this simplest approximation provides reasonable results, at least in cases where no strong structural rearrangement occurs. To improve the description, it is possible to move to more sophisticated vibronic coupling models, which are readily created using established protocols. ${ }^{26,28}$

For the purpose of advancing to larger, more complex systems it will be possible to use embedding methods, to proceed through interpolation of diabatic Hamiltonians ${ }^{19,20}$ or to incorporate exciton models in the dynamics. ${ }^{98-100}$

\section{Conflicts of interest}

There are no conflicts to declare.

\section{Acknowledgements}

Funding from the University of Vienna and the Austrian Science Fund (FWF) within project I2883 is gratefully acknowledged. FP thanks Loughborough University for startup funding. The Vienna Scientific Cluster is thanked for computational support. MM gratefully acknowledges financial support from the EU Horizon 2020 research and innovation program under the Marie SkłodowskaCurie grant agreement no. 642294. The authors thank M. Fumanal, E. Gindensperger, C. Daniel, and G. A. Worth for discussions regarding the LVC model.

\section{References}

1 J. C. Tully, J. Chem. Phys., 1990, 93, 1061-1071.

2 D. Polli, P. Altoè, O. Weingart, K. M. Spillane, C. Manzoni, D. Brida, G. Tomasello, G. Orlandi, P. Kukura, R. A. Mathies, M. Garavelli and G. Cerullo, Nature, 2010, 467, 440-443.

3 M. Barbatti, A. J. A. Aquino, J. J. Szymczak, D. Nachtigallová, P. Hobza and H. Lischka, Proc. Natl. Acad. Sci. U. S. A., 2010, 107, 21453-21458.

4 S. Gozem, H. L. Luk, I. Schapiro and M. Olivucci, Chem. Rev., 2017, 117, 13502-13565.

5 G. Cui and W. Thiel, Angew. Chem., Int. Ed., 2013, 52, 433-436. 6 A. V. Akimov, A. J. Neukirch and O. V. Prezhdo, Chem. Rev., 2013, 113, 4496-4565.

7 M. Richter, P. Marquetand, J. Gonzalez-Vazquez, I. Sola and L. Gonzalez, J. Chem. Theory Comput., 2011, 7, 1253-1258.

8 S. Mai, M. Pollum, L. Martínez-Fernández, N. Dunn, P. Marquetand, I. Corral, C. E. Crespo-Hernández and L. González, Nat. Commun., 2016, 7, 13077.

9 C. Xu, L. Yu, C. Zhu, J. Yu and Z. Cao, Sci. Rep., 2016, 6, 26768. 10 J. P. Zobel, J. J. Nogueira and L. González, Chem. - Eur. J., 2018, 24, 5379-5387.
11 A. J. Atkins and L. González, J. Phys. Chem. Lett., 2017, 8, 3840-3845.

12 M. Barbatti, WIREs Comput. Mol. Sci., 2011, 1, 620.

13 T. Nelson, S. Fernandez-Alberti, A. E. Roitberg and S. Tretiak, Acc. Chem. Res., 2014, 47, 1155-1164.

14 L. Du and Z. Lan, J. Chem. Theory Comput., 2015, 11, 1360-1374.

15 S. Pal, D. J. Trivedi, A. V. Akimov, B. Aradi, T. Frauenheim and O. V. Prezhdo, J. Chem. Theory Comput., 2016, 12, 1436-1448.

16 J. E. Subotnik, A. Jain, B. Landry, A. Petit, W. Ouyang and N. Bellonzi, Annu. Rev. Phys. Chem., 2016, 67, 387-417.

17 S. Mai, P. Marquetand and L. González, WIREs Comput. Mol. Sci., 2018, e1370.

18 R. Crespo-Otero and M. Barbatti, Chem. Rev., 2018, 118, 7026-7068.

19 J. W. Park and Y. M. Rhee, J. Chem. Theory Comput., 2014, 10, 5238-5253.

20 J. W. Park and Y. M. Rhee, J. Chem. Phys., 2014, 140, 164112.

21 J. Ischtwan and M. A. Collins, J. Chem. Phys., 1994, 100, 8080-8088.

22 G. W. Richings, I. Polyak, K. E. Spinlove, G. A. Worth, I. Burghardt and B. Lasorne, Int. Rev. Phys. Chem., 2015, 34, 269-308.

23 J. Behler and M. Parrinello, Phys. Rev. Lett., 2007, 98, 146401.

24 M. Gastegger, J. Behler and P. Marquetand, Chem. Sci., 2017, 8, 6924-6935.

25 H. Köppel, W. Domcke and L. S. Cederbaum, Adv. Chem. Phys., 1984, 57, 59-246.

26 H. Köppel, J. Gronki and S. Mahapatra, J. Chem. Phys., 2001, 115, 2377-2388.

27 M. Fumanal and C. Daniel, J. Comput. Chem., 2016, 3, 2454-2466.

28 C. Cattarius, A. Markmann and G. A. Worth, The VCHAM program, 2007, see http://www.pci.uni-heidelberg.de/tc/ usr/mctdh/doc.85/vcham/index.html.

29 G. A. Worth and L. S. Cederbaum, Annu. Rev. Phys. Chem., 2004, 55, 127-158.

30 M. H. Beck, A. Jäckle, G. A. Worth and H.-D. Meyer, Phys. Rep., 2000, 324, 1-105.

31 A. Raab, G. A. Worth, H.-D. Meyer and L. S. Cederbaum, J. Chem. Phys., 1999, 110, 936-946.

32 R. P. Krawczyk, K. Malsch, G. Hohlneicher, R. C. Gillen and W. Domcke, Chem. Phys. Lett., 2000, 320, 535-541.

33 S. Faraji, H.-D. Meyer and H. Köppel, J. Chem. Phys., 2008, 129, 074311.

34 C. Lévêque, A. Komainda, R. Taïeb and H. Köppel, J. Chem. Phys., 2013, 138, 044320.

35 G. A. Worth, G. Welch and M. J. Paterson, Mol. Phys., 2006, 104, 1095-1105.

36 J. Eng, C. Gourlaouen, E. Gindensperger and C. Daniel, Acc. Chem. Res., 2015, 48, 809-817.

37 H. Tamura and I. Burghardt, J. Am. Chem. Soc., 2013, 135, 16364-16367.

38 U. Müller and G. Stock, J. Chem. Phys., 1997, 107, 6230-6245.

39 S. Mai, P. Marquetand and L. González, Int. J. Quantum Chem., 2015, 115, 1215-1231. 
40 S. Mai, M. Richter, M. Heindl, M. F. S. J. Menger, A. Atkins, M. Ruckenbauer, F. Plasser, M. Oppel, P. Marquetand and L. González, Sharc2.0: Surface Hopping Including Arbitrary Couplings - Program Package for Non-Adiabatic Dynamics, 2018, available from http://sharc-md.org.

41 C. Xie, X. Hu, L. Zhou, D. Xie and H. Guo, J. Chem. Phys., 2013, 139, 014305.

42 I. Wilkinson, A. E. Boguslavskiy, J. Mikosch, J. B. Bertrand, H. J. Wörner, D. M. Villeneuve, M. Spanner, S. Patchkovskii and A. Stolow, J. Chem. Phys., 2014, 140, 204301.

43 S. Mai, P. Marquetand and L. González, J. Chem. Phys., 2014, 140, 204302.

44 C. Lévêque, R. Taïeb and H. Köppel, J. Chem. Phys., 2014, 140, 091101.

45 C. Lévêque, D. Peláez, H. Köppel and R. Taïeb, Nat. Commun., 2014, 5, 4126.

46 F. Franco de Carvalho and I. Tavernelli, J. Chem. Phys., 2015, 143, 224105.

47 J. Heicklen, N. Kelly and K. Partymiller, Res. Chem. Intermed., 1980, 3, 315-404.

48 C. R. Guest, R. A. Hochstrasser, L. C. Sowers and D. P. Millar, Biochemistry, 1991, 30, 3271-3279.

49 L. Serrano-Andres, M. Merchan and A. C. Borin, Proc. Natl. Acad. Sci. U. S. A., 2006, 103, 8691-8696.

50 S. Ullrich, T. Schultz, M. Z. Zgierski and A. Stolow, Phys. Chem. Chem. Phys., 2004, 6, 2796.

51 N. L. Evans and S. Ullrich, J. Phys. Chem. A, 2010, 114, 11225-11230.

52 M. Barbatti and H. Lischka, J. Am. Chem. Soc., 2008, 130, 6831-6839.

53 E. Fabiano and W. Thiel, J. Phys. Chem. A, 2008, 112, 6859-6863.

54 A. N. Alexandrova, J. C. Tully and G. Granucci, J. Phys. Chem. B, 2010, 114, 12116-12128.

55 S. Lobsiger, S. Blaser, R. K. Sinha, H. M. Frey and S. Leutwyler, Nat. Chem., 2014, 6, 989-993.

56 T. Fiebig, C. Wan and A. H. Zewail, ChemPhysChem, 2002, 3, 781-788.

57 M. Pollum, L. Martínez-Fernández and C. E. CrespoHernández, in Photochemistry of Nucleic Acid Bases and Their Thio- and Aza-Analogues in Solution, ed. M. Barbatti, A. C. Borin and S. Ullrich, Springer International Publishing, Cham, 2015, pp. 245-327.

58 S. Mai, M. Pollum, L. Martínez-Fernández, N. Dunn, P. Marquetand, I. Corral, C. E. Crespo-Hernández and L. González, Nat. Commun., 2016, 7, 1-8.

59 D. Koyama, M. J. Milner and A. J. Orr-Ewing, J. Phys. Chem. B, 2017, 121, 9274-9280.

60 S. Bai and M. Barbatti, Phys. Chem. Chem. Phys., 2017, 19, 12674-12682.

61 J. H. Saiki, K. B. McCredie, T. J. Vietti, J. S. Hewlett, F. S. Morrison, J. J. Costanzi, W. J. Stuckey, J. Whitecar and B. Hoogstraten, Cancer, 1978, 42, 2111-2114.

62 T. Kobayashi, H. Kuramochi, Y. Harada, T. Suzuki and T. Ichimura, J. Phys. Chem. A, 2009, 113, 12088-12093.

63 A. C. Borin, S. Mai, P. Marquetand and L. González, Phys. Chem. Chem. Phys., 2017, 19, 5888-5894.
64 S. Mai, F. Plasser, P. Marquetand and L. González, Attosecond Molecular Dynamics, The Royal Society of Chemistry, 2019.

65 M. Fumanal, F. Plasser, S. Mai, C. Daniel and E. Gindensperger, J. Chem. Phys., 2018, 148, 124119.

66 M. S. Schuurman and D. R. Yarkony, J. Chem. Phys., 2007, 127, 094104.

67 H. Lischka, M. Dallos, P. G. Szalay, D. R. Yarkony and R. Shepard, J. Chem. Phys., 2004, 120, 7322-7329.

68 T. Ichino, J. Gauss and J. F. Stanton, J. Chem. Phys., 2009, 130, 174105.

69 A. Tajti and P. G. Szalay, J. Chem. Phys., 2009, 131, 124104.

70 F. Plasser, M. Ruckenbauer, S. Mai, M. Oppel, P. Marquetand and L. González, J. Chem. Theory Comput., 2016, 12, 1207.

71 G. Granucci, M. Persico and A. Toniolo, J. Chem. Phys., 2001, 114, 10608-10615.

72 G. Granucci, M. Persico and A. Zoccante, J. Chem. Phys., 2010, 133, 134111.

73 F. Plasser, G. Granucci, J. Pittner, M. Barbatti, M. Persico and H. Lischka, J. Chem. Phys., 2012, 137, 22 A514.

74 P. G. Szalay, T. Muller, G. Gidofalvi, H. Lischka and R. Shepard, Chem. Rev., 2012, 112, 108.

75 B. O. Roos, R. Lindh, P.-Å. Malmqvist, V. Veryazov and P.-O. Widmark, J. Phys. Chem. A, 2004, 108, 2851-2858.

76 M. Reiher, Theor. Chem. Acc., 2006, 116, 241-252.

77 B. A. Heß, C. M. Marian, U. Wahlgren and O. Gropen, Chem. Phys. Lett., 1996, 251, 365-371.

78 P. A. Malmqvist, B. O. Roos and B. Schimmelpfennig, Chem. Phys. Lett., 2002, 357, 230-240.

79 S. Mai, T. Müller, F. Plasser, P. Marquetand, H. Lischka and L. González, J. Chem. Phys., 2014, 141, 074105.

80 H. Lischka, M. Dallos and R. Shepard, Mol. Phys., 2002, 100, 1647-1658.

81 R. A. Kendall, T. H. Dunning Jr. and R. J. Harrison, J. Chem. Phys., 1992, 96, 6796.

82 H. Lischka, R. Shepard, R. M. Pitzer, I. Shavitt, M. Dallos, T. Muller, P. G. Szalay, M. Seth, G. S. Kedziora, S. Yabushita and Z. Y. Zhang, Phys. Chem. Chem. Phys., 2001, 3, 664-673.

83 H. Lischka, T. Müller, P. G. Szalay, I. Shavitt, R. M. Pitzer and R. Shepard, Wiley Interdiscip. Rev.: Comput. Mol. Sci., 2011, 1, 191-199.

84 H. Lischka, R. Shepard, I. Shavitt, R. M. Pitzer, M. Dallos, T. Muller, P. G. Szalay, F. B. Brown, R. Ahlrichs, H. J. Boehm, A. Chang, D. C. Comeau, R. Gdanitz, H. Dachsel, C. Ehrhardt, M. Ernzerhof, P. Hoechtl, S. Irle, G. Kedziora, T. Kovar, V. Parasuk, M. J. M. Pepper, P. Scharf, H. Schiffer, M. Schindler, M. Schueler, M. Seth, E. A. Stahlberg, J.-G. Zhao, S. Yabushita, Z. Zhang, M. Barbatti, S. Matsika, M. Schuurmann, D. R. Yarkony, S. R. Brozell, E. V. Beck, J.-P. Blaudeau, M. Ruckenbauer, B. Sellner, F. Plasser, R. F. K. Szymczak, J. J. Spada and A. Das, COLUMBUS: An Ab Initio Electronic Structure Program, Release 7.0, www.univie. ac.at/columbus, 2017.

85 F. Aquilante, et al., J. Comput. Chem., 2016, 37, 506-541.

86 T. Helgaker, H. J. A. Jensen, P. Jorgensen, J. Olsen, K. Ruud, H. Agren, T. Andersen, K. L. Bak, V. Bakken, 
O. Christiansen, P. Dahle, E. K. Dalskov, T. Enevoldsen, H. Heiberg, H. Hettema, D. Jonsson, S. Kirpekar, R. Kobayashi, H. Koch, K. V. Mikkelsen, P. Norman, M. J. Packer, T. Saue, P. R. Taylor and O. Vahtras, DALTON, an $a b$ initio electronic structure program, release 1.0, 1997.

87 J. P. Dahl and M. Springborg, J. Chem. Phys., 1988, 88, 4535-4547.

88 TURBOMOLE V7.0.1 2017, a development of University of Karlsruhe and Forschungszentrum Karlsruhe $\mathrm{GmbH}$, 1989-2007, TURBOMOLE GmbH, since 2007, available from http://www.turbomole.com.

89 S. Nangia, A. W. Jasper, T. F. Miller and D. G. Truhlar, J. Chem. Phys., 2004, 120, 3586-3597.

90 D. Golomb, K. Watanabe and F. F. Marmo, J. Chem. Phys., 1962, 36, 958-960.

91 J. Bloino, M. Biczysko, F. Santoro and V. Barone, J. Chem. Theory Comput., 2010, 6, 1256-1274.

92 M. Barbatti, Z. Lan, R. Crespo-Otero, J. J. Szymczak, H. Lischka and W. Thiel, J. Chem. Phys., 2012, 137, 22 A503.
93 C. Plützer, E. Nir, M. S. de Vries and K. Kleinermanns, Phys. Chem. Chem. Phys., 2001, 3, 5466-5469.

94 A. Holmén, B. Nordén and B. Albinsson, J. Am. Chem. Soc., 1997, 119, 3114-3121.

95 K. A. Seefeld, C. Plützer, D. Löwenich, T. Häber, R. Linder, K. Kleinermanns, J. Tatchen and C. M. Marian, Phys. Chem. Chem. Phys., 2005, 7, 3021.

96 D. Picconi, F. J. Avila Ferrer, R. Improta, A. Lami and F. Santoro, Faraday Discuss., 2013, 163, 223.

97 F. Plasser, R. Crespo-Otero, M. Pederzoli, J. Pittner, H. Lischka and M. Barbatti, J. Chem. Theory Comput., 2014, 10, 1395-1405.

98 R. Binder, J. Wahl, S. Römer and I. Burghardt, Faraday Discuss., 2013, 163, 205.

99 A. Sisto, C. Stross, M. W. van der Kamp, M. O’Connor, S. McIntosh-Smith, G. T. Johnson, E. G. Hohenstein, F. R. Manby, D. R. Glowacki and T. J. Martinez, Phys. Chem. Chem. Phys., 2017, 19, 14924-14936.

100 S. Jurinovich, L. Cupellini, C. A. Guido and B. Mennucci, J. Comput. Chem., 2018, 39, 279-286. 